\title{
Superior sensation: superior colliculus participation in rat vibrissa system
}

\author{
Marie E Hemelt ${ }^{1,2}$ and Asaf Keller*2
}

Address: ${ }^{1}$ Program in Neuroscience, University of Maryland School of Medicine, Baltimore, MD, USA and ${ }^{2}$ Department of Anatomy \& Neurobiology, University of Maryland School of Medicine, Baltimore, MD, USA

Email: Marie E Hemelt - mpohl002@umaryland.edu; Asaf Keller* - akeller@umaryland.edu

* Corresponding author

Published: 3I January 2007

BMC Neuroscience 2007, 8:12 doi:10.1 |86/147|-2202-8-12

This article is available from: http://www.biomedcentral.com/I47I-2202/8/I2

(C) 2007 Hemelt and Keller; licensee BioMed Central Ltd.

This is an Open Access article distributed under the terms of the Creative Commons Attribution License (http://creativecommons.org/licenses/by/2.0), which permits unrestricted use, distribution, and reproduction in any medium, provided the original work is properly cited.
Received: 27 October 2006

Accepted: 31 January 2007

\begin{abstract}
Background: The superior colliculus, usually considered a visuomotor structure, is anatomically positioned to perform sensorimotor transformations in other modalities. While there is evidence for its potential participation in sensorimotor loops of the rodent vibrissa system, little is known about its functional role in vibrissa sensation or movement. In anesthetized rats, we characterized extracellularly recorded responses of collicular neurons to different types of vibrissa stimuli.

Results: Collicular neurons had large receptive fields (median $=14.5$ vibrissae). Single units displayed responses with short latencies $(5.6 \pm 0.2 \mathrm{msec}$, median $=5.5)$ and relatively large magnitudes ( $1.2 \pm 0.1$ spikes/stimulus, median $=1.2$ ). Individual neurons could entrain to repetitive vibrissa stimuli delivered at $\leq 20 \mathrm{~Hz}$, with little reduction in phase locking, even when response magnitude was decreased. Neurons responded preferentially to vibrissa deflections at particular angles, with $43 \%$ of the cells having high $(\geq 5)$ angular selectivity indices.

Conclusion: Results are consistent with a proposed role of the colliculus in somatosensorymediated orienting. These properties, together with the connections of the superior colliculus in sensorimotor loops, are consistent with its involvement in orienting, alerting and attentive functions related to the vibrissa system.
\end{abstract}

\section{Background}

The superior colliculus has long been studied as a center for visual sensory and motor responses [1-3], and is involved in orienting attention [4-6] and multimodal processing [7-10]. The functions of the colliculus have been characterized principally in relation to vision and eye movements, whereas its role in other sensorimotor transformations has received less attention. We seek to explore the function of the superior colliculus using the vibrissa system, a vital sensorimotor system for the rat. Although previous studies demonstrated the existence of vibrissa-responsive collicular neurons $[9,11-13]$, there have been no systematic, quantitative analyses of their response properties to controlled stimuli. The goal of this study was to correct this deficiency.

The superior colliculus is thought to be part of a sensorimotor loop in the rat vibrissa system, receiving input from trigeminal nuclei and projecting to the vibrissa motoneurons [see [14]]. In addition, the superior colliculus interacts with other structures of the vibrissa-barrel system: it receives input from the barrel cortex and from the vibrissa area of the motor cortex [15-17], and receives inputs from the cerebellum and basal ganglia [see [18]]. It is recipro- 
cally connected with the zona incerta [19], a structure recently shown to be involved in state dependent gating of vibrissal inputs in the thalamus [20-22]. Because of these anatomical relationships, and because of their role in orienting responses, we hypothesized that collicular neurons respond with short latency and strong directional selectivity to vibrissal stimuli.

\section{Results \\ Location of vibrissa responsive units}

We report data from 51 vibrissa-responsive units recorded from 24 animals. We used histological analyses, stereotaxic coordinates and depth readings from the manipulator to determine the location of the recorded units. Due to the size of the lesions, we did not separate intermediate gray and white layers, but divided the colliculus into superficial, intermediate, and deep layers. Vibrissa responsive units were located in the intermediate layers of the lateral and posterior parts of the colliculus. Stereotaxic coordinates were 2.2 to $2.6 \mathrm{~mm}$ lateral and 7.6 to $7.9 \mathrm{~mm}$ posterior to Bregma, at a depth of 3.2 to 5.3 below the cortical surface. Clusters of vibrissa responsive neurons were interspersed with clusters responding to manual stimulation of other body parts. Vibrissa-responsive clusters ranged in size from 100 to 300 microns along the axis of penetration; it was not possible to reliably determine the cluster size in the horizontal plane without fine-grained mapping in a single animal. The clustering of vibrissa responses may reflect the clustering in the colliculus of afferents from the trigeminal nuclei $[23,24]$.

\section{Waveform morphology}

Neurons in several brain regions have been classified according to their action potential waveforms [25,26]. For example, cortical vibrissa-responsive units include regular and fast spiking neurons $[27,28]$. In extracellular recordings, inhibitory, fast spiking neurons have action potentials with a brief ( $\leq 180 \mu \mathrm{sec})$ negativity followed by a brief positivity ( $\leq 400 \mu \mathrm{sec})$, whereas regular spiking neurons have longer waveform components (> $180 \mu \mathrm{sec}$ and $>400 \mu \mathrm{sec}$ ). The superior colliculus contains both excitatory (glutamatergic) and inhibitory (GABAergic) neurons, which may segregate into similar categories [18,29]. To test this, we measured the two components of their extracellularly recorded waveforms, and plotted the positive component against the negative component (Fig. 1). All data points (51 data points, 12 overlapping) formed a single cluster with values encompassing those of cortical regular and fast spiking units. While we cannot exclude the possibility that our sample included a heterogeneous population of neurons, these findings suggest that our recordings could not distinguish neuronal subclasses based on their waveforms.

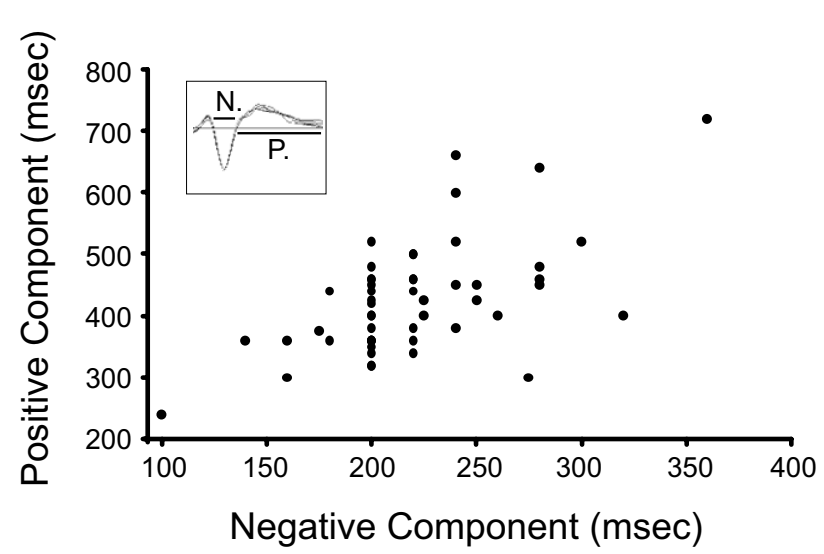

Figure I

Neuronal subclasses could not be distinguished by their waveform morphology. The inset shows representative waveforms, with the negative $(\mathrm{N})$ and positive $(\mathrm{P})$ components indicated. When the positive component was plotted against the negative component, data points formed a single cluster.

\section{Spontaneous activity}

Vibrissa-responsive neurons had low or no spontaneous activity (e.g. Fig. 2A-C; 44/48 neurons had no spontaneous activity, mean was $0.01 \pm 0.006 \mathrm{~Hz}$ ). Therefore, to avoid biasing our recording to spontaneously active units, we searched for units while manually stimulating the vibrissae. We did find many spontaneously active, albeit non-responsive units near these relatively quiescent, responsive neurons. We found no neurons for which stimulation evoked a decrease in spontaneous activity.

\section{Response latency}

The superior colliculus receives input from a variety of structures that process vibrissa inputs, including the trigeminal nuclear complex and the neocortex (see Background). We reasoned that if collicular responses represent direct inputs from the trigeminal nuclei, these responses would have relatively short latencies, similar to those of thalamic neurons that receive trigeminal inputs. Figures 2A-B depict responses recorded from two representative collicular neurons, showing the latency of their responses to vibrissa stimuli delivered as an air-puff (Fig. 2A; 6.4 msec onset latency) or delivered with the piezoelectric device (Fig. 2B; $7.0 \mathrm{msec}$ ). For consistency, here and in subsequent analyses (magnitude, duration, and response ratios), we report responses to the vibrissa deflections in the caudal direction, the direction mimicking most vibrissa contacts during active whisking. When using the piezoelectric device, we stimulated the vibrissa evoking the largest response. 


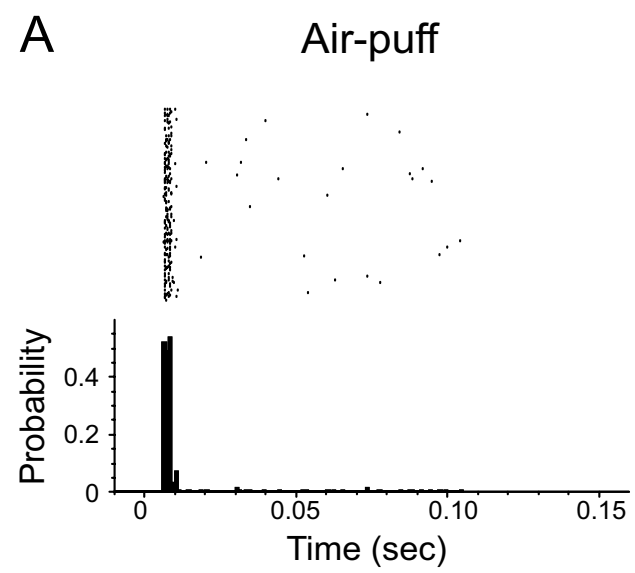

B Piezo
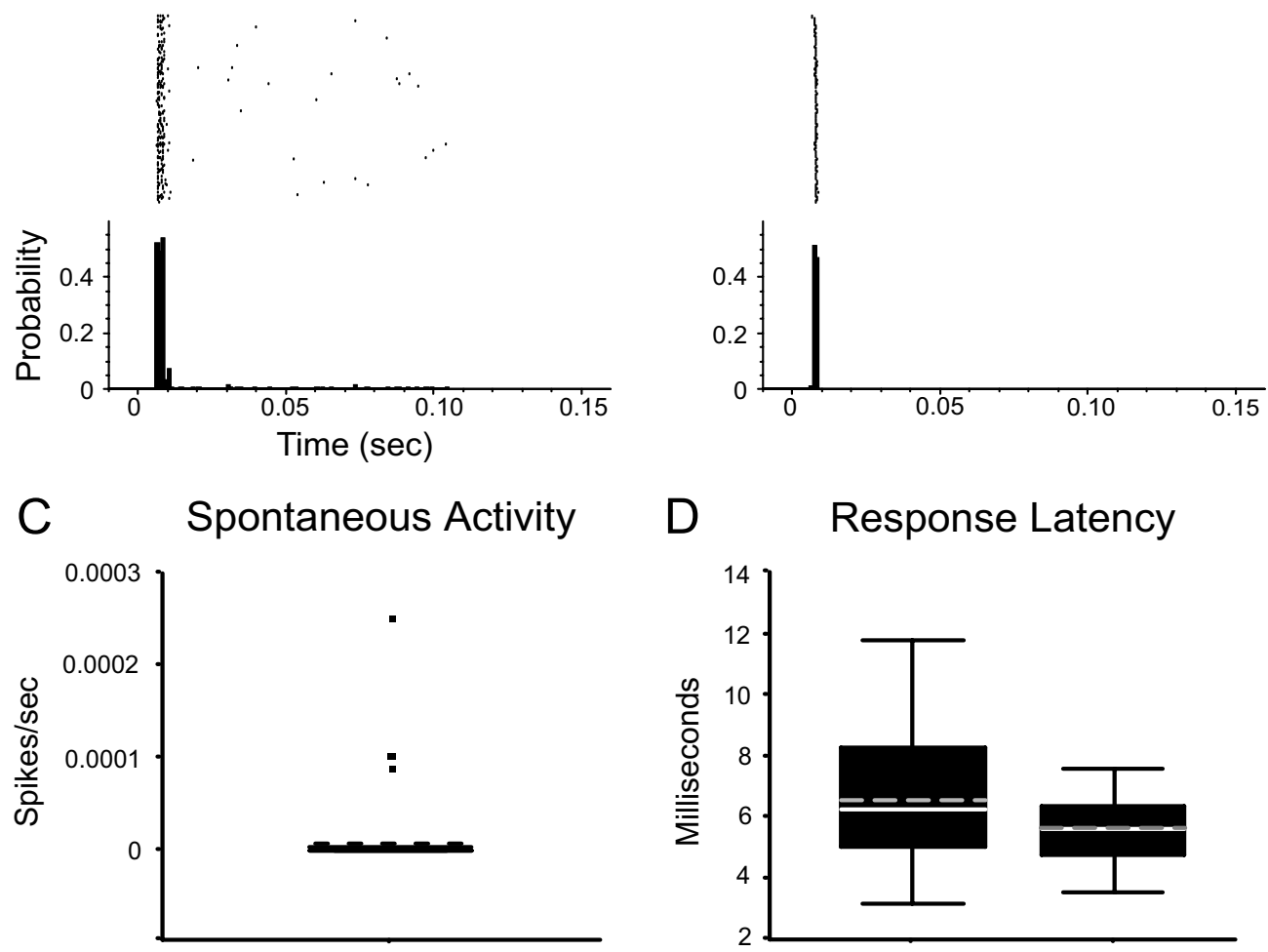

D Response Latency

$\mathrm{E}$

Response Magnitude
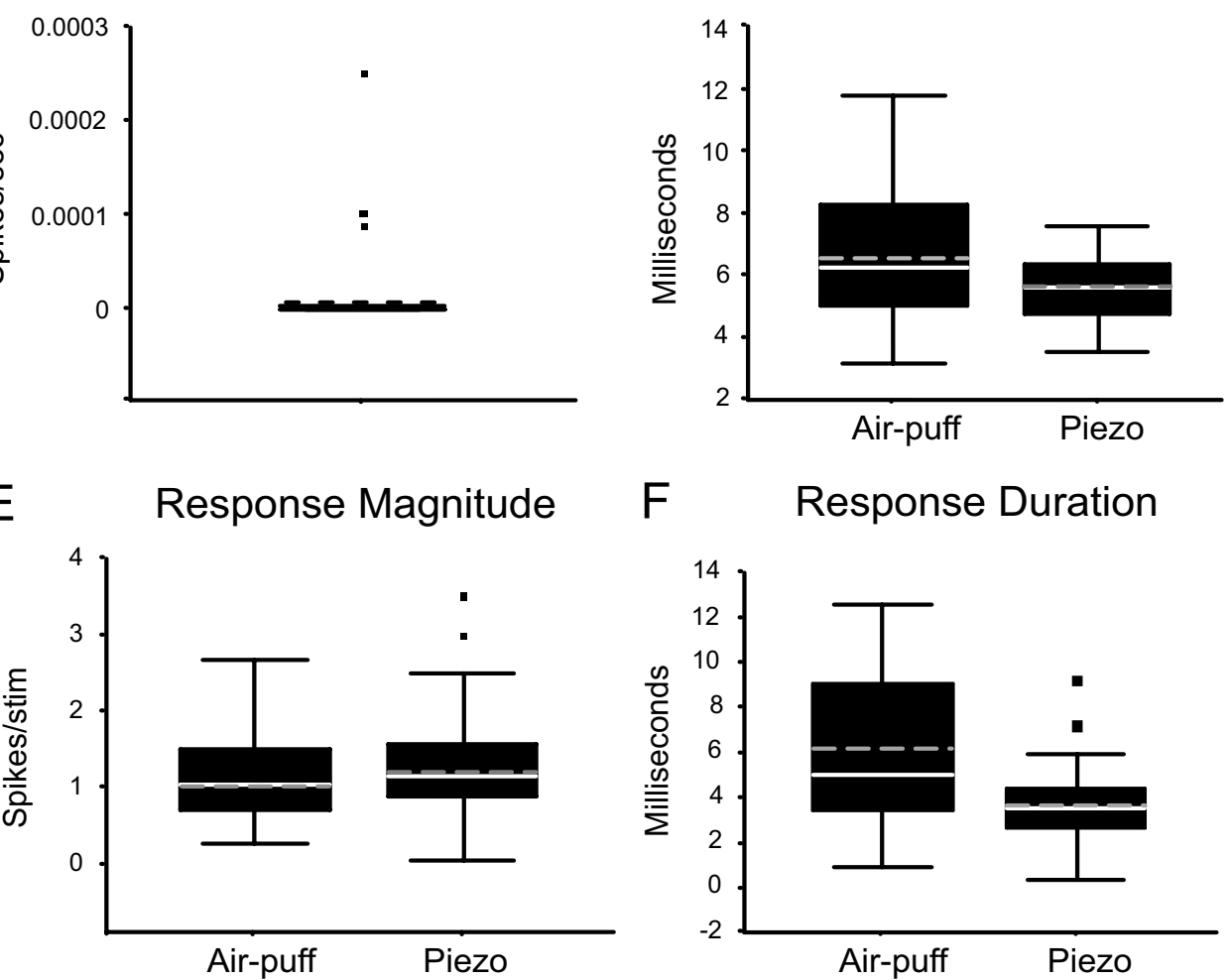

F Response Duration

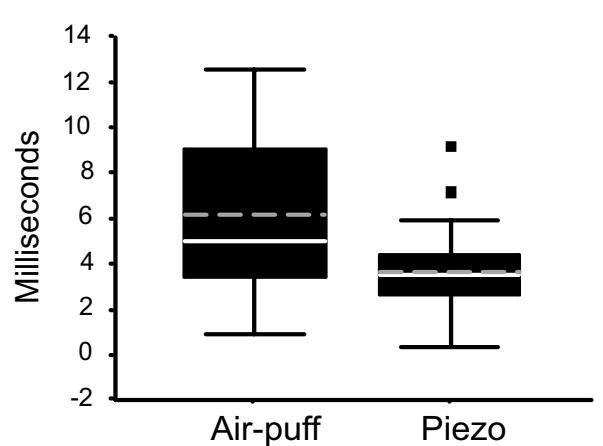

Figure 2

Response kinetics of superior colliculus neurons. A-C: PSTHs of representative responses constructed with I msec bins. Collicular responses to air-puffs $(A)$ or piezoelectric (B, piezo) vibrissa deflections. C-F: Boxplots depict median (solid line), mean (dotted line) and $\mid$ st and $3^{\text {rd }}$ quartiles (box). Whiskers represent data within 1.5 times the range from the $\mid$ st to the $3^{\text {rd }}$ quartile. Squares represent outliers (data outside I.5 times the range from the Ist to the $3^{\text {rd }}$ quartile). C: Neurons in the superior colliculus have low or no spontaneous activity. $D$ : Response latencies evoked by air-puff deflections were not significantly different than those evoked by piezoelectric deflections $(P=0.2)$. E: Response magnitude was unaffected by stimulus type $(P=$ $0.7)$. F: Response durations were short and unaffected by stimulus type $(P=0.07)$. All statistical comparisons use the Kolmogorov-Smirnov test. 
Group data are depicted in the boxplots in Figure 2D. Piezoelectric stimulation of a single vibrissa elicited a response with median latency of $5.5 \mathrm{msec}(5.6 \pm 0.2 \mathrm{msec}$, $\mathrm{n}=31$ ). In response to air-puff stimulation of multiple vibrissae, the median latency was $6.2 \mathrm{msec}(6.5 \pm 0.6, \mathrm{n}=$ $15)$. There is no significant difference in latency between these two groups $(P=0.2)$. Thus, collicular neurons display short latency responses to vibrissa inputs, similar to their counterparts in the ventrobasal thalamus.

\section{Response kinetics}

Ramp-and-hold stimuli, delivered with the piezoelectric device, evoked phasic responses in all 31 neurons tested, with robust spiking only at the onset $(\mathrm{ON})$ and offset (OFF) of the stimuli (Fig. 3A, 200 msec stimuli; Fig. 3B, 30 msec stimuli). The durations of ON responses evoked with piezoelectric deflections were very short, with all 31 neurons showing durations less than $10 \mathrm{msec}$ (26 less than $5 \mathrm{msec}, 3.7 \pm 0.4$, Fig. 2F). Air-puff stimuli produced more temporally dispersed responses (e.g., Fig. 2A), and, as a result, response duration was more varied but not significantly different from the piezoelectric evoked responses (range 1.0-12.6 msec, $6.2 \pm 0.92 \mathrm{msec}, \mathrm{n}=16$, $P=0.07)$. Using criteria introduced by Simons and Carvell [30] we classified all the neurons tested as rapidly adapting (responses lasting $<50 \mathrm{msec}$ ).

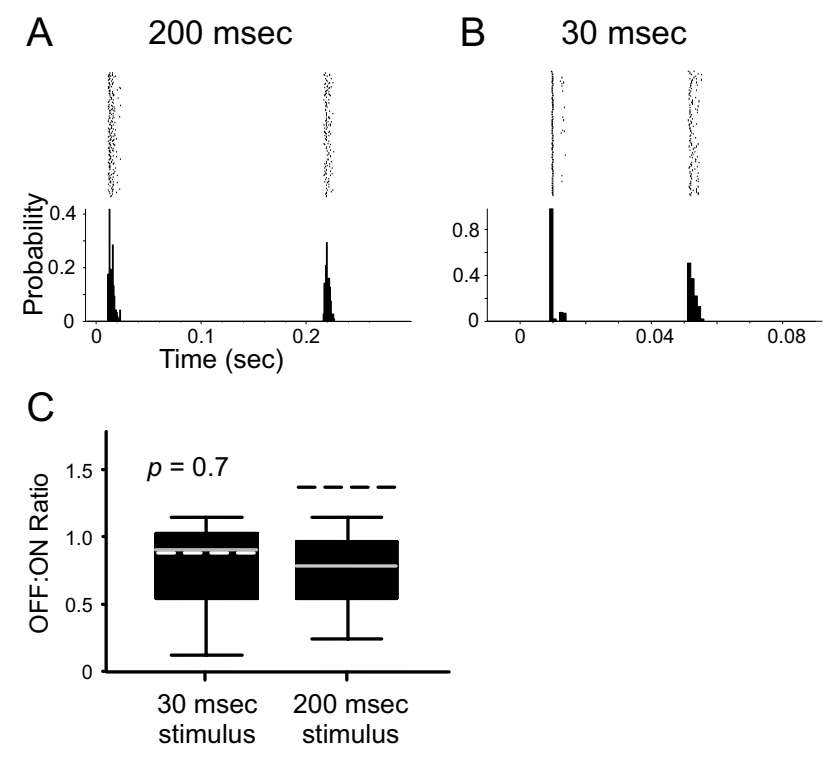

\section{Figure 3}

OFF:ON response magnitude ratios for vibrissaresponsive neurons. Examples of responses to $200 \mathrm{msec}$ stimuli (A) and 30 msec stimuli (B) in different neurons. C: Boxplots depicting OFF:ON ratios for 200 and $30 \mathrm{msec} s t i m-$ uli. Decreasing the stimulus duration had little effect on the OFF:ON ratio $(P=0.7)$.
Mean response magnitudes to caudal vibrissa deflections (spikes per stimulus, see Methods) were $1.1 \pm 0.1$ for airpuff stimulation $(\mathrm{n}=16)$, and $1.2 \pm 0.1$ for piezoelectric stimulation ( $n=31$, Fig. 2E). We also tested the response to stimuli in the preferred direction (the direction evoking the largest response); piezoelectric stimulation produced a mean response magnitude of $1.6 \pm 0.2$ (median $1.3, \mathrm{n}=$ 23). The angular selectivity of these neurons is described in detail below.

In both cortex and thalamus, OFF response magnitudes are generally smaller than ON response magnitudes [31]. When we applied a $200 \mathrm{msec}$ caudal deflection with the piezoelectric device, the OFF:ON ratio was $1.4 \pm 0.5(0.5$ $\mathrm{n}=20$ where deflection evoked both a detectable ON and OFF response), larger than that reported previously in barrel cortex $[0.55 \pm 0.03,[31]]$. When the stimulus duration was decreased to $30 \mathrm{msec}$ (e.g. Fig. 3B), the OFF response decreased somewhat, though the evoked OFF:ON ratio was statistically indistinguishable from that of the longer deflection (Fig. 3C, ratio $=0.9 \pm 0.1, P=0.3, \mathrm{n}=13$, unpaired t-test). Thus, changing stimulus duration had no significant effect on OFF:ON response magnitude ratios.

\section{Receptive field size}

Receptive field size varies along the vibrissa-barrel neural axis, from a single vibrissa in the trigeminal ganglion to a median of nine vibrissae in SII cortex [28,32]. We tested the receptive field of 36 neurons by manually deflecting individual vibrissae, and monitoring the responses on an oscilloscope and through an audio amplifier. Figure 4B shows the distribution of receptive field sizes for collicular neurons $($ median $=14.5$ vibrissae, $13.8 \pm 0.1)$. Vibrissae encompassing the receptive field of a particular neuron were always located contiguously on the whisker pad (e.g. Fig 4A). These large receptive fields are compared below to others in the vibrissa-barrel axis.

\section{Principal vibrissa analysis}

In the barrel cortex, neurons responding to stimulation of more than one vibrissa respond preferentially to the vibrissa associated with their anatomically defined barrel. This vibrissa is referred to as the principal whisker, and its stimulation evokes a response with larger magnitude and shorter onset latency than stimulation of other vibrissae in the receptive field $[26,28]$. We examined both response magnitude and latency as potential metrics for distinguishing principal whiskers in the superior colliculus.

We examined responses to individual vibrissae within a receptive field using piezoelectric deflections. In cases where unit isolation degraded before the experiment was completed, it was not possible to test all the vibrissae associated with a particular neuron. Because of the smaller amplitude of vibrissa deflections produced with the pie- 

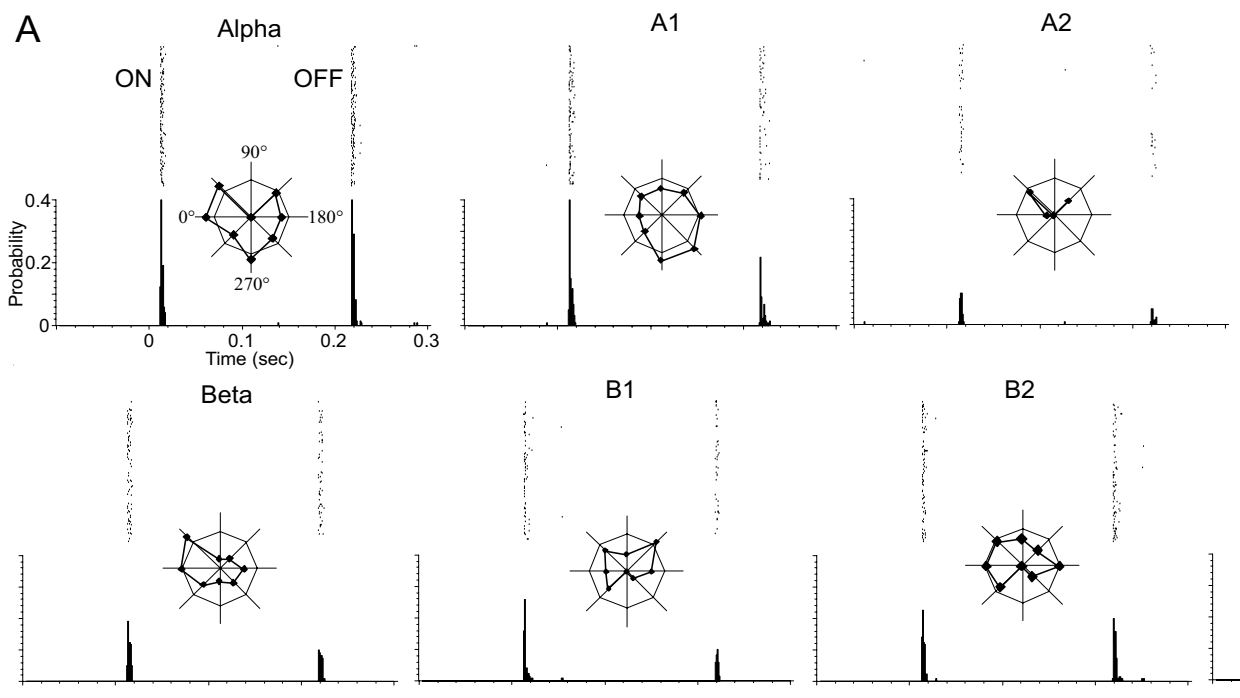

B2

B3

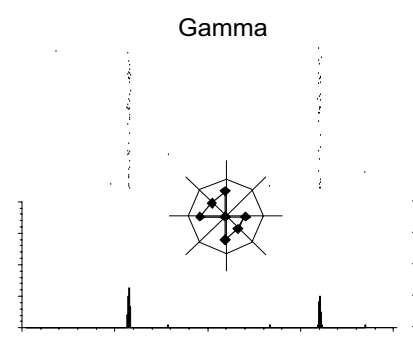

C1
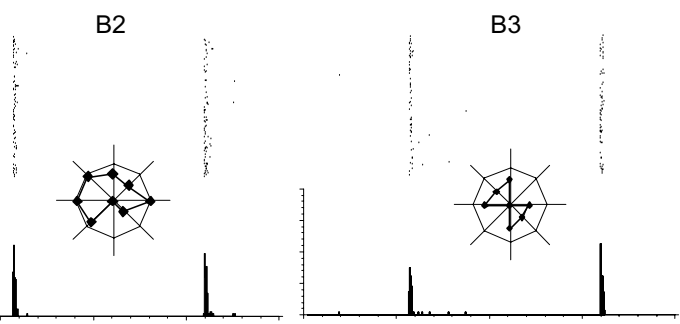

C2

B

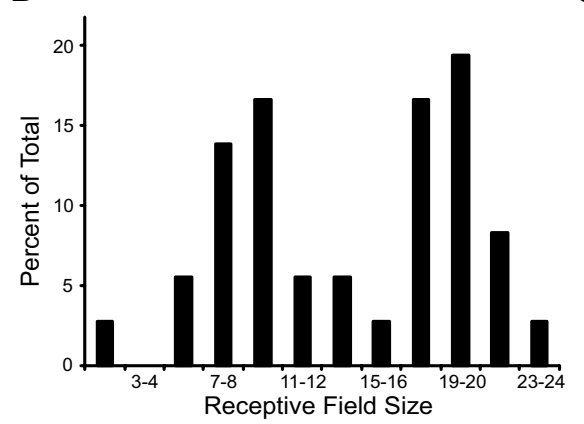

C

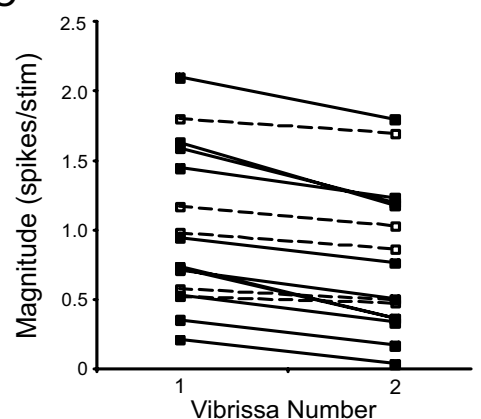

D

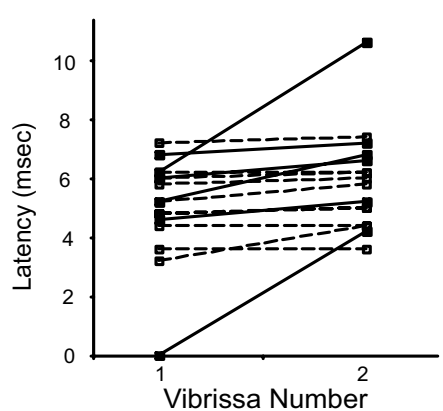

Figure 4

Superior colliculus neurons have large receptive fields with principal whiskers. A: An example of a neuron with a I7vibrissa receptive field. Responses to individual deflections of 10 vibrissae with the piezoelectric device are depicted, with the vibrissa deflected indicated above each PSTH. Vibrissae were deflected in eight different directions $\left(0^{\circ}\right.$ is caudal, $90^{\circ}$ is dorsal), and $\mathrm{ON}$ responses to each direction were used to create the polar tuning curves. Distance from the center of the polar tuning curve represents the magnitude of the response for deflections in that direction. The radii of the octagons represent one spike/ stimulus. The PSTHs were constructed by pooling spikes recorded in response to each of the eight directions. $B$ : Histogram of receptive field sizes of collicular neurons, defined as the number of vibrissae evoking responses to manual stimulation. $C$ : Difference in response magnitude to stimulation of different vibrissae. For each neuron, vibrissae were ordered according to their response magnitudes ("VI" evoked the largest magnitude response, "V2" the second largest magnitude, etc). Eleven of I6 neurons showed a significant difference in magnitude between VI and V2 (solid lines, paired t-test, $P<0.005$ ). D: Difference in onset latency to stimulation of different vibrissae. For each neuron, vibrissae were ordered according to their response latency ("VI" evoked responses at the shortest latency, etc). Six of 16 neurons showed a significant difference in latency between $\mathrm{VI}$ and V2 (solid lines, paired t-test, $\mathrm{P}<0.005$ ). 
zoelectric device, not all the vibrissae within the receptive field-defined with manual stimulation-produced significant responses to piezoelectric stimuli.

Figure 4A shows responses of a single neuron to deflections of 10 different vibrissae within a 17 -vibrissa receptive field (A3, B4, C3, C4, C5, $\gamma$ and D5 were not tested with piezoelectric stimulation). Each vibrissa was deflected at eight different angles, pseudo-randomly, with the piezoelectric device. In these polar plots, the distance from the origin represents the magnitude of the ON response for deflections at that angle; the angular selectivity of this neuron is discussed below. The overall response of the neuron to each vibrissa was obtained by pooling responses to all eight directions and constructing PSTHs, shown here below the polar plots. Because different whiskers within a receptive field did not have the same preferred direction, we used these PSTHs, which contain information from all eight deflection angles, for the analysis of response magnitude and latency as metrics for determining a principle whisker. The representative neuron in Figure 4A responded to each of the vibrissae with similar magnitude and latency.

This analysis was performed on 16 neurons for which we tested at least 5 vibrissae. For each neuron, we rankordered the vibrissae according to the magnitude of evoked responses. The vibrissa evoking the largest response magnitude was defined as V1, the next largest as $\mathrm{V} 2$, etc. We compared response magnitudes for V1 and V2 using a t-test. These response magnitudes were significantly different in $69 \%$ of neurons $(11$ of $16, \mathrm{P}<0.005)$ indicating that deflection of this vibrissa evoked a larger magnitude response than any other vibrissa in the receptive field.

In addition, we rank-ordered these vibrissae according to the latency of evoked responses (the shortest latency being V1, etc). Although the response latencies increase somewhat (Fig 4D), we found only 6 of 16 neurons $(38 \%)$ where the increase was significant between the V1 and V2. This indicates that V1 did not always evoke a response significantly faster than the vibrissa evoking responses at the next shortest latency. In eight of 16 neurons, the vibrissa evoking the largest magnitude response was also the vibrissa with the shortest latency.

Thus, collicular neurons respond to multiple vibrissae with similar latency but with different response magnitudes. This indicates that superior colliculus neurons have a principal whisker, despite their large receptive fields. Responses to non-principal (adjacent) vibrissae continue to be robust: For example, the vibrissae ranked V5 evoked a response of $0.4 \pm 0.1$ spikes/stimulus $(n=14)$, a value that was $44 \%$ of the principal whisker response. For con- sistency, in other analyses we focused on the vibrissa evoking the largest response, that is, the principal whisker.

\section{Angular tuning characteristics}

Neurons throughout the vibrissa-to-cortex pathway of the rat are selective for the angle of vibrissa deflection $[26,28,30,33,34]$, eliciting larger magnitude responses to preferred angle deflections. The strength of neuronal angular preference varies along this neuroaxis; for example, thalamocortical neurons have stronger angular selectivity than barrel neurons [30]. To test the angular preference of collicular neurons, we deflected vibrissae in eight different directions pseudo-randomly, for a total of 120 deflections ( 15 in each direction, e.g. Fig. 5A). Figure $5 \mathrm{~B}$ presents a polar tuning curve from a neuron with strong angular preference, and Figure 5C depicts results from a poorly tuned neuron. In these tuning curves, the distance from the origin represents the magnitude of the ON response to a deflection at that angle, with $0^{\circ}$ representing the caudal direction and $90^{\circ}$ representing the dorsal direction.

To quantify angular preference we determined, for each neuron-vibrissa pair, the number of deflection angles evoking an $\mathrm{ON}$ response magnitude that was statistically smaller than the response to the maximally activating angle (compared using Student's t-test, $P<0.005$ ). We then categorized tuning curves into eight groups ( 0 to 7 ) representing the number of angles with significantly smaller responses. Category 0 represents the least-tuned responses (equal response to all deflection angles) and category 7 represents the best-tuned responses (respond preferentially to one deflection angle). Figure 5D shows the distribution of selectivity indices for 23 neurons, computed from responses to the vibrissa evoking the largest magnitude response. The mean selectivity index was 3.4, with well-tuned cells (categories 5 to 7 ) constituting 43\% of these neuron-vibrissa pairs.

When we included in this analysis responses to all vibrissae (and not only to the vibrissa maximally activating the neuron), the distribution of selectivity indices shifted to less-tuned values (29\% well-tuned, 126 vibrissa-neurons pairs, Fig 5E). This is because these vibrissae evoked smaller responses, many of which were statistically indistinguishable from zero. As a result, the contrast between responses to deflections at different angles was reduced. We therefore applied a second metric, the angular tuning ratio [35], computed by dividing the response magnitude in the preferred direction by the overall response magnitude. Although this metric does not allow for statistical comparisons, it is less sensitive to zero responses in a particular direction. This ratio was $1.7 \pm 0.1($ median $=1.5)$ for vibrissa producing the largest response in each neuron, and $2.2 \pm 0.1$ (median $=1.7$ ) for all vibrissa-neuron pairs. 

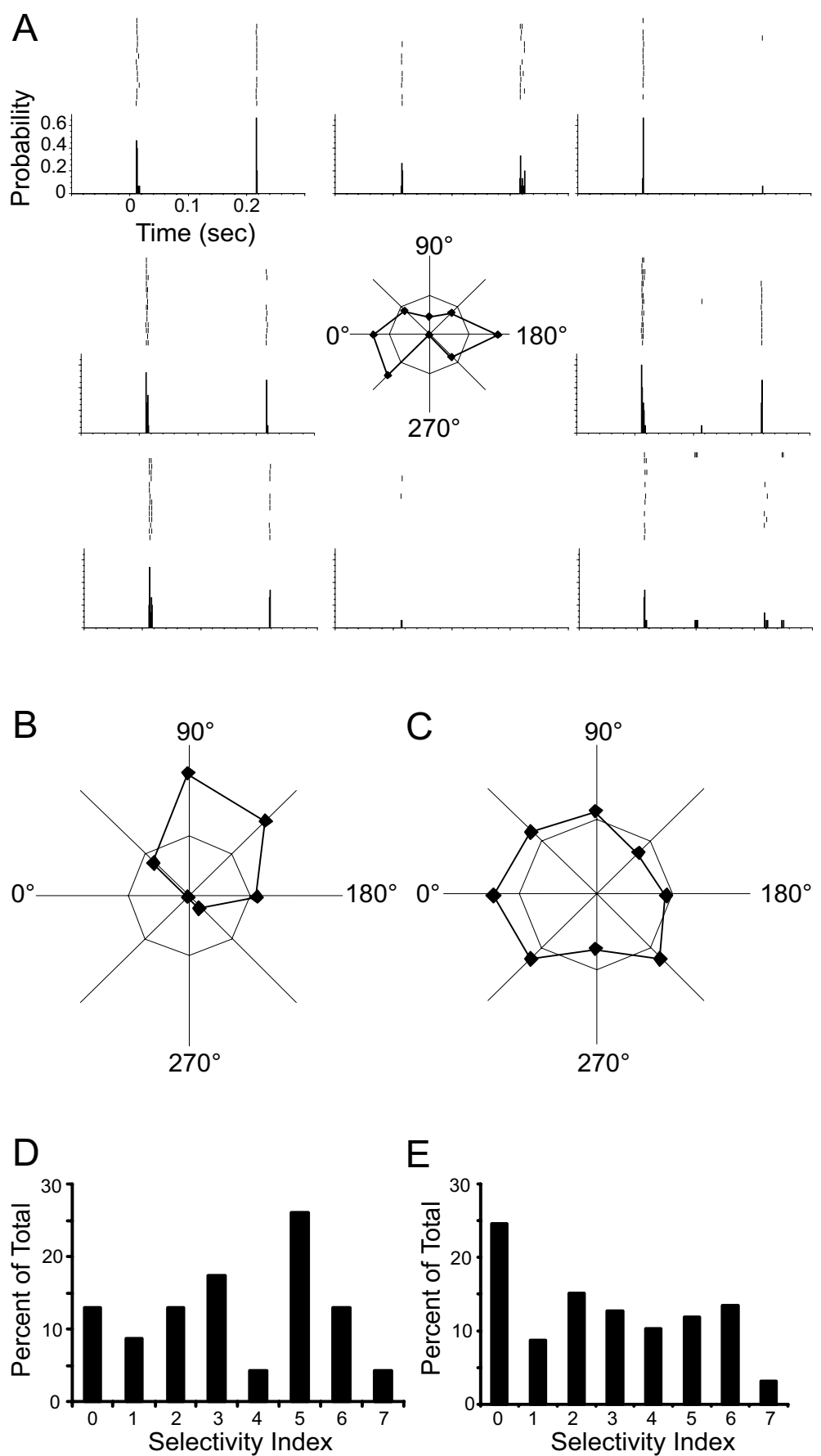

Figure 5

Superior colliculus neurons display a high degree of angular selectivity. A: To determine angular selectivity, vibrissae were deflected in eight directions. ON responses in each direction were used to compute the selectivity index (SI) and create the polar tuning curve (center). Distance from the center in the polar tuning curve represents the magnitude of the response for deflections in that direction. Octagon radii represent one spike/stimulus. B: A well-tuned neuron $(\mathrm{SI}=5)$. $C$ : $A$ poorly tuned neuron $(\mathrm{SI}=\mathrm{I})$. Histograms in $D$ and $E$ depict the SIs for all neurons tested, either considering only responses to the vibrissa evoking the largest response in each neuron $(D)$; or considering all vibrissa-neuron pairs $(E)$. 
Thus, both the selectivity index and tuning ratio metrics indicate that collicular neurons respond preferentially to vibrissa deflections at particular angles.

\section{Response adaptation}

We tested the ability of collicular neurons to respond to a range of stimulation frequencies $(0.5$ to $20 \mathrm{~Hz}$, e.g. Fig. 6A-D). As stimulation frequency increased, response magnitude decreased for both air-puff and piezoelectric stimulation (Fig. 6E; air-puff: 1, 2, 5, 8, 11, and $20 \mathrm{~Hz}, \mathrm{P}$ $=0.028, \mathrm{n}=10$; piezoelectric: $0.5,1,2,5,8,11$, and $15 \mathrm{~Hz}$, $\mathrm{P}<0.001, \mathrm{n}=11$; Friedman test). There were no significant differences between magnitudes of responses to piezoelectric or air-puff stimulation at any stimulation frequency $(\mathrm{P}>1.3,20 \mathrm{~Hz}$ air-puff was compared to $15 \mathrm{~Hz}$ piezoelectric). Response magnitude at the highest frequencies tested $(15 \mathrm{~Hz}$ or $20 \mathrm{~Hz}$ ) was $0.2 \pm 0.07$ spikes/ stimulus for air-puff stimulation, and $0.6 \pm 0.2$ for piezoelectric stimulation.

Even when response magnitudes are reduced, phase locking of a response to a stimulus can be important in transmitting information. To test phase locking of collicular neurons at different frequencies, we computed the coefficient of variation (CV) of response latencies, using only the first spike of each response. Because the piezoelectric device generates the most temporally accurate stimulation onset, we analyze only responses to this device. The average CV for $0.5 \mathrm{~Hz}$ stimulation was $0.04 \pm 0.006$. The CV decreased significantly as stimulation frequency increased (Fig. 6F, $P=0.007$, Friedman's test). The mean $\mathrm{CV}$ did not exceed $0.1( \pm 0.02)$, a value reached at the highest stimulus frequency $(15 \mathrm{~Hz})$ capable of producing linear responses from the piezoelectric device. These findings indicate that, even in response to high frequency stimulation, when response magnitude is decreased, the entrainment of collicular neurons is still appreciable.

\section{Discussion}

\section{Spontaneous activity}

The spontaneous activity of these neurons is quite low. Values $(0.08 \pm 0.06 \mathrm{~Hz})$ are lower than those recorded in the ventroposterior medial thalamus [VPM, $14.2 \mathrm{~Hz}$ under halothane, 7.9 Hz under pentobarbital, [30,36]] and somatosensory cortex $[1.1 \mathrm{~Hz},[30]]$. This is unlikely due to a general anesthesia-induced suppression of spontaneous activity, as we found many neurons with high rates of spontaneous activity in all layers of the colliculus. The low rate of spontaneous activity in vibrissa-responsive units suggests that this pathway is equipped to respond with high signal-to-noise ratios.

\section{Receptive fields}

The receptive fields of collicular neurons are unique compared to those in other areas of the vibrissa-barrel path- way. Receptive fields are large $($ median $=14.5,13.8 \pm 0.1)$, with none of the cells responding only to a single vibrissa. Receptive fields in barrel cortex tend to be smaller, with $55 \%$ of neurons responding only to a single vibrissa [26]. The VPM nucleus of the thalamus contains neurons which respond to an average of three vibrissae, $[ \pm 1,[37]]$, receptive fields that are considerably smaller that those of collicular neurons. Collicular receptive fields are also larger than those in the second somatosensory cortex, previously characterized as having unusually large receptive fields [median $=9$, [28]]. In the paralemniscal POm (posterior nucleus of the thalamus), where receptive fields are also large, usually only stimulation of many vibrissae can evoke a response [38,39]. By contrast, all collicular neurons were capable of responding to deflections of an individual vibrissa. A principal whisker could be identified based on response magnitude, while responses to adjacent vibrissae were also robust. The broad receptive fields we observed could be an artifact of anesthesia. However, anesthesia tends to sharpen vibrissa receptive fields, and not to broaden them $[40,41]$.

\section{Angular preference}

Collicular neurons are more selective to the angle of vibrissa deflection, compared to neurons in VPM or barrel cortex: 43 percent of collicular neurons have a selectivity index of 5 or greater, whereas only $31 \%$ of VPM cells and $16 \%$ of barrel cortex neurons have an equally high selectivity index [30]. The superior colliculus receives its main trigeminal input from the trigeminal sub-nucleus interpolaris (SpVi), and angular tuning of collicular neurons may reflect the properties of these input neurons, which contains a population of highly tuned neurons [42]. However, this tuning is not always consistent across the receptive field of collicular neurons (e.g., Fig. 3A). This may be a result of convergence from neurons in $\mathrm{SpVi}$ that are tuned to different directions.

\section{Response kinetics}

Collicular neurons respond to whisker deflections at short latencies $(5.9 \pm 0.4 \mathrm{msec})$. These latencies are similar to those previously reported in VPM $[6.4 \pm 1.8 \mathrm{msec},[43]])$, and are shorter than those in POm [27.4 $\pm 2.2 \mathrm{msec}[22]]$. Thus, consistent with anatomical findings (see Background), this suggests that collicular neurons are receiving input directly from the trigeminal nucleus, and are not being activated by cortical or thalamic loops.

Because the superior colliculus receives its main trigeminal input from the SpVi, it is considered part of the paralemniscal system [44]. However, the colliculus displays more robust responses than $\mathrm{POm}$, which also receives direct input from SpVi. Responses in POm are characterized by their long latency (see above) and low magnitude [0.7 \pm 0.1 spikes/stim, [22]], compared to collicular 

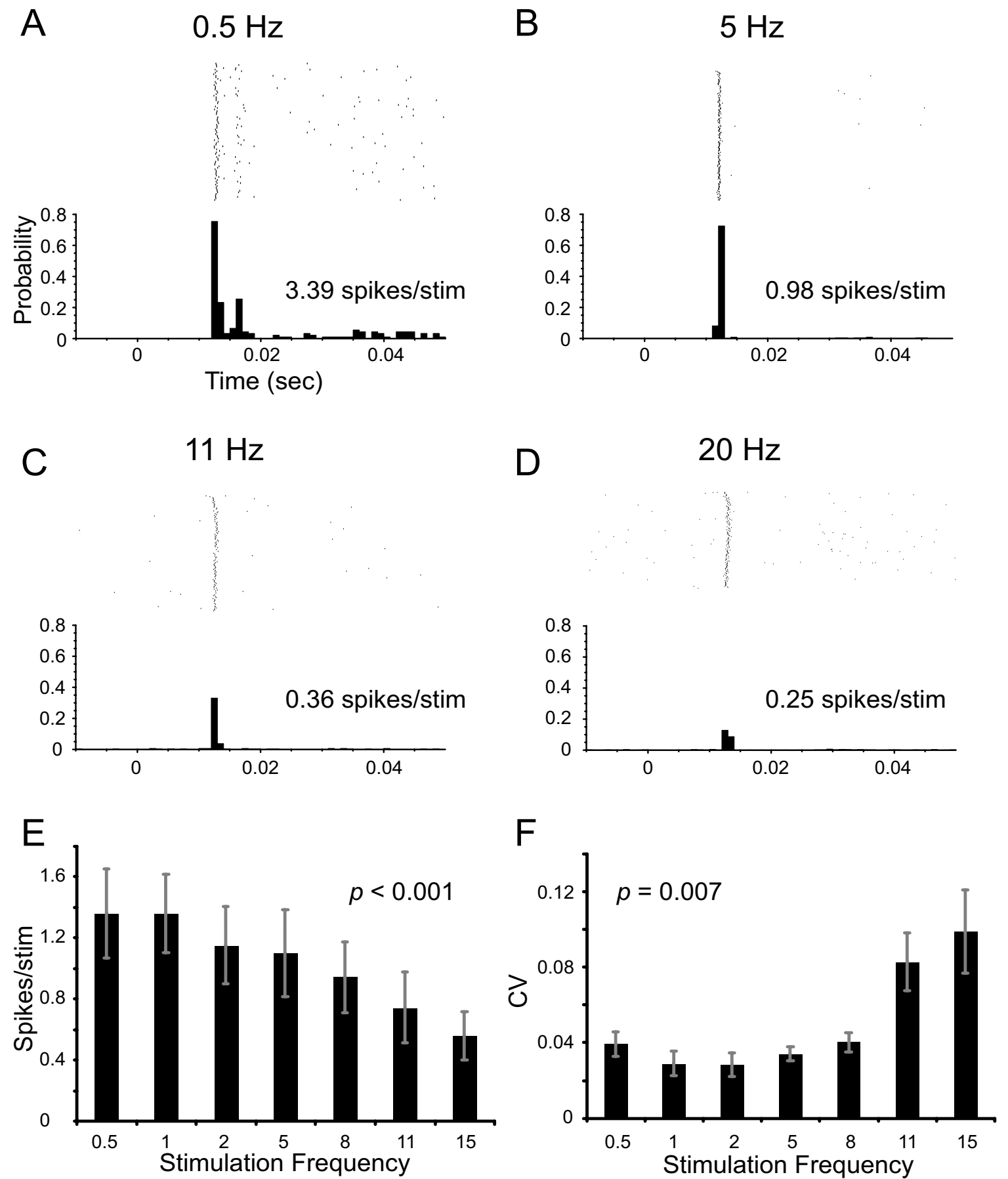

\section{Figure 6}

Response adaptation in the superior colliculus. PSTHs (A-D) show response characteristics of a single cell over a range of stimulation frequencies. Significant response magnitudes are indicated for each PSTH. E: Response magnitude decreases significantly as stimulation frequency increases (Friedman's test, $P<0.00 \mathrm{I}$ ). F: Phase-locking, measured as the coefficient of variation $(C V)$ of the latency, also decreases significantly with increasing frequency stimulation $(P=0.007)$, but the $C V$ remains relatively low even at high frequency. 
responses $(1.9 \pm 0.4$ spikes/stim). Additionally, neurons in the paralemniscal system, including POm, typically respond poorly at increasing stimulation frequencies $[38,45]$. Collicular neurons, in comparison, respond to 15 $\mathrm{Hz}$ stimulation with $38 \%$ of the response magnitude evoked by $0.5 \mathrm{~Hz}$ stimulation (0.6 spikes/stim compared to 1.6 spikes/stim). However, POm responses are enhanced when inhibition from zona incerta to POm is suppressed $[20,22]$. It remains to be determined if the colliculus, which is reciprocally connected with zona incerta [19], is also regulated by inhibition from this structure.

Even when inhibition from zona incerta is intact, collicular responses are large (median $=1.2$ spikes $/ \mathrm{stim}$ ), and similar to those seen at several levels of the lemniscal system: 0.99 spikes/stimulus in VPM [46] and 1.8 spikes/ stimulus in barrel cortex [28]. The superior colliculus does receive some input from the trigeminal nucleus principalis $[44,47]$, and these inputs could be driving these robust collicular responses.

The ratio of rapidly adapting to slowly adapting neurons decreases along the vibrissa-to-cortex neuroaxis. This reduction is greater in the paralemniscal system. The proportion of slowly adapting neurons in the lemniscal system is relatively large: $93 \%$ in the principal trigeminal nucleus [34], 37\% in VPM, and 15\% in barrel cortex [30]. The paralemniscal pathway begins with a smaller proportion of slowly adapting neurons, with $54 \%$ of the cells in SPVi [48] and only $3 \%$ of the neurons in the second somatosensory cortex [28]. In the superior colliculus, none of the neurons we recorded from were slowly adapting, lending further support to the assumption that the colliculus is part of the paralemniscal system.

OFF:ON response magnitude ratios in the superior colliculus $(1.4 \pm 0.5)$ are larger than those of excitatory barrel cortex neurons $(0.55 \pm 0.03,29)$. Experimental and modeling studies indicate that smaller OFF:ON ratios in barrel cortex are a consequence of the dominance of inhibition in the layer IV damping circuit [31]. The larger OFF:ON ratios in the colliculus may reflect a lesser role for inhibition in this structure, at least under our experimental conditions. Whereas inhibition in barrel cortex may be important for spatiotemporal discrimination, lesser inhibition in the superior colliculus may optimize this structure for alerting and orienting functions.

Collicular neurons had decreased response magnitude and increased CV in response to higher frequency stimuli (Fig. 6F). Nevertheless, collicular neurons displayed precise phase locking at all frequencies tested, with low average coefficient of variance for the latency (from 0.04 at 0.5 $\mathrm{Hz}$ to 0.1 at $15 \mathrm{~Hz}$ ). These values are much smaller than those reported in POm, which ranged from 0.58 to 0.82 over 0.5 to $11 \mathrm{~Hz}$ [38]. Thus, collicular neurons respond to high-frequency stimulation with more temporal precision than neurons in other parts of the paralemniscal system. The high response fidelity of superior colliculus neurons demonstrates that this structure is well equipped for accurate frequency discrimination.

The high OFF:ON ratios and robust, time-locked responses indicate that the superior colliculus is well suited for the accurate detection of a novel or relevant stimuli. Although collicular neurons entrain to repetitive stimuli, response probability decreases as the cells habituate (Fig. 6). This suggests that non-novel stimuli may result in reduced output from collicular circuits. These issues need to be addressed in unanesthetized, behaving animals.

\section{Conclusion}

Several aspects of the neuronal response properties discussed here are consistent with superior colliculus involvement in orienting responses and alerting or attentive functions related to somatosensation in the rat. The low levels of spontaneous activity create the potential for high signal-to-noise levels both in collicular processing and in efferent signaling. The short latency, large magnitude, and reliability of the responses are behaviorally advantageous for rapid and consistent awareness of novel or relevant stimuli. The apparent minor role of inhibition, as evidenced in the large OFF:ON ratios, may also be optimized for alerting functions. The interconnections of the superior colliculus with many other structures involved in sensation (thalamus, cortex) and arousal (zona incerta) suggests this structure may play a role in regulating awareness of vital stimuli.

\section{Methods}

\section{Surgical procedures}

We performed experiments using 24 female SpragueDawley rats weighing 200 to $350 \mathrm{~g}$. All procedures strictly adhered to institutional and federal guidelines. We maintained the rats within anesthetic stages III-3 to IV [40] with isoflurane (1.0 to $2.0 \%$ ), administered through a tracheal tube. Body temperature was maintained at $37^{\circ} \mathrm{C}$ with a servo-controlled heating blanket. Following infusion of local anesthetics at surgical sites, we performed a craniotomy ( $1.5 \mathrm{~mm}$ diameter) over the cortex covering the superior colliculus.

\section{Recording}

We obtained extracellular unit recordings with either quartz-insulated platinum electrodes or glass-insulated tungsten electrodes (both 2 to $4 \mathrm{M} \Omega$ ). Electrodes were advanced perpendicular to the cortical surface, using either an Inchworm stepper motor (Burleigh, Fishers, NY), or a multi-electrode manipulator (Alpha Omega, 
Israel or Thomas Recording, Germany). We continuously stimulated vibrissae on the contralateral face during electrode penetrations to detect units with low or no spontaneous activity. Waveforms recorded from well-isolated units were digitized at $40 \mathrm{kHz}$ through an AlphaLab data acquisition system (Alpha Omega). We isolated units offline with a combination of threshold and waveform component analysis using Off-Line Sorter (Plexon, Dallas). We report data from 48 single units and 3 multiunit recordings; we combined data from multiunits and single units as these sets were indistinguishable.

Recording sites were marked with electrolytic lesions ( 5 to $10 \mu \mathrm{A}$ for $20 \mathrm{sec})$. The animals were deeply anesthetized with sodium pentobarbital $(60 \mathrm{mg} / \mathrm{kg})$ and perfused transcardially with buffered saline followed by $4 \%$ buffered paraformaldehyde. Recording sites were identified in Nissl-stained coronal sections.

\section{Vibrissa stimulation}

We determined the size of receptive fields by manually deflecting individual vibrissae. Two types of controlled vibrissa stimulation were used: A piezoelectric device that deflected a single vibrissa, or an air-puff stimulus that deflected several vibrissae. Vibrissae deflected individually were inserted into a small tube-approximately $10 \mathrm{~mm}$ from their base-attached to a computer-controlled piezoelectric stimulator that can be deflected in eight different directions (courtesy of Dr. D.J. Simons, Univ. Pittsburgh). We applied ramp-and-hold stimuli, either $200 \mathrm{~ms}$ or 30 msec in duration, having onset/offset velocity of $102 \mathrm{~mm} /$ $\mathrm{s}$. The shorter stimulus duration was used to compare responses to different frequencies of repeated stimuli (see below). To reduce mechanical ringing, the trapezoid ramp-and-hold waveforms were filtered with a Bessel filter. The peak onset and offset velocity were measured as the slope of the linear portion of the deflection ramp. We calibrated the stimulator with a photodiode device.

The piezoelectric stimulator was used to compare responses both to different frequencies of stimulation and to different angles of deflection. In the frequency paradigm, all deflections were in the caudal direction at varying frequencies $(0.5,1,2,5,8,11$ or $15 \mathrm{~Hz})$. In the angular paradigm the vibrissa was deflected in eight different directions (in $45^{\circ}$ increments). In these experiments, deflections were at $1 \mathrm{~Hz}$ with a pseudo random sequence for a total of 15 stimuli per deflection angle (120 deflections total).

Air-puff stimuli deflecting several vibrissae were delivered through a tube $(0.5 \mathrm{~mm}$ diameter $)$ by a Picospritzer ${ }^{\mathrm{rm}}$ (General Valve, Fairfield, NJ). We delivered air-puffs at $0.5,1,2,5,8,11$ or $20 \mathrm{~Hz}$, with a pressure of $60 \mathrm{psi}$, resulting in vibrissa deflections of approximately $30^{\circ}$. The dura- tion of the air-puff was $200 \mathrm{msec}$, except when testing the response to different frequency stimuli, when the air-puff duration was reduced to $30 \mathrm{msec}$ (for $20 \mathrm{~Hz}$ stimuli) or 50 msec (for all other frequencies).

In our analyses of response kinetics, we included data on responses to stimulation at the lowest frequency tested for each neuron $(0.5 \mathrm{~Hz}, 1 \mathrm{~Hz}$, or $2 \mathrm{~Hz})$. There were no significant differences in any response kinetic tested in response to these different frequencies.

\section{Data analysis}

Time stamps of well-isolated units and of stimulus triggers were exported to Matlab (MathWorks, Natick, MA) for analyses using custom written software. Peristimulus time histograms (PSTHs, $0.2 \mathrm{msec}$ bins) were constructed from these time stamps. For display purposes, PSTHs in the figures were constructed using $1 \mathrm{msec}$ bins. Significant stimulus-evoked responses were defined as PSTH bins whose response magnitude significantly exceeded $(99 \%$ confidence interval) spontaneous activity levels, computed from a baseline period preceding each stimulus. Response onset was defined as the occurrence of two consecutive post-stimulus bins $(0.4 \mathrm{msec})$ displaying significant responses. Response offset was defined as 10 consecutive bins $(5 \mathrm{msec})$ in which no significant response occurred. We used these criteria to objectively and consistently define significant response epochs, relative to baseline firing.

Statistical analyses were performed in SPSS and Microsoft Excel. Where appropriate, results are displayed using a boxplot to depict the median and distribution of the data (see Fig. 2D). Between-group statistical comparisons were assessed with the nonparametric Kolmogorov-Smirnov (K-S) test, because it is sensitive to any type of distributional differences (i.e., central tendency, variability, skewness, and kurtosis) and makes no assumptions regarding normality or equivalence of variance. Tests for withingroup comparisons are specified in the text. Data are presented as median or mean \pm standard error.

\section{Authors' contributions}

MEH participated in designing the study, carried out electrophysiological experiments and performed the analysis. $\mathrm{AK}$ conceived and directed the project. Both authors drafted the manuscript and have approved the final manuscript.

\section{Acknowledgements}

Supported by PHS: NINDS grants NS-05 I799 and NS-31078.

\section{References}

I. McHaffie JG, Stein BE: Eye movements evoked by electrical stimulation in the superior colliculus of rats and hamsters. Brain Res 1982, 247:243-253. 
2. Hanes D, Wurtz R: Interaction of the frontal eye field and superior colliculus for saccade generation. J Neurophysiol 200I, 85(2):804-8I5.

3. Brecht $M$, Singer $W$, Engel $A$ : Amplitude and direction of saccadic eye movements depend on the synchronicity of collicular population activity. J Neurophysiol 2004, 92:424-432.

4. Sahibzada N, Dean P, Redgrave P: Movements resembling orientation or avoidance elicited by electrical stimulation of the superior colliculus in rats. J Neurosci 1986, 6(3):723-733.

5. Muller J, Philiastides M, Newsome W: Microstimulation of the superior colliculus focusses attention without moving the eyes. Proc Natl Acad Sci USA 2004, I 02(3):524-529.

6. Goldberg W, Wurtz R: Activity of superior colliculus in behaving monkey. II. Effect of attention on neuronal responses. Neurophysiol 1972, 35(4):560-574.

7. Stein B, Wallace M, Stanford T: Development of multisensory integration: transforming sensory input into motor output. Mental Retardation and Developmental Disabilities 1999, 5:72-85.

8. Wallace M, Meredith M, Stein B: Multisensory integration in the superior colliculus of the alert cat. I Neurophysiol 1998, 80:1006-1010.

9. Stein B, Magalhaes-Castro B, Kruger L: Relationship between visual and tactile representations in cat superior colliculus. Neurophysiol 1976, 39(2):40I-4I9.

10. Cavanaugh J, Alvarez BD, Wurtz RH: Enhanced performance with brain stimulation: attentional shift or visual cue? I Neurosci 2006, 26(44): I I347-I I358.

II. Drager U, Hubel D: Responses to visual stimulation and relationship between visual, auditory, and somatosensory inputs in mouse superior colliculus. J Neurophysiol 1975, 38(3):690-713.

12. Benedetti F: Orienting behaviour and superior colliculus sensory representations in mice with the vibrissae bent into the contralateral hemispace. Eur J Neurosci 1995, 7(7):1512-1519.

13. Rhoades RW, Mooney R, Jacquin M: Complex somatosensory receptive fields of cells in the deep laminae of the hamster's superior colliculus. J Neurosci 1983, 3(7): 1342-1354.

14. Kleinfeld D, Berg RW, O'Conner SM: Anatomical loops and their electrical dynamics in relation to whisking by rat. Somatosens Motor Res 1999, 16(2):69-88.

15. Miyashita E, Keller A, Asanuma H: Input-output organization of the rat vibrissae motor cortex. Exp Brain Res 1994, 99:223-232.

16. Hattox AM, Priest CA, Keller A: Functional circuitry involved in the regulation of whisker movements. J Comp Neurol 2002, 442(3):266-276

17. Miyashita E, Mori S: The superior colliculus relays signals descending from the vibrissal motor cortex to the facial nerve nucleus in the rat. Neurosci Lett 1995, 195:69-71.

18. May P: The mammalian superior colliculus: laminar structure and connections. Progress in Brain Research 2005, 15 1:32 I-378.

19. Gregory U, Hall W: Pathway from the zona incerta to the superior colliculus in the rat. J Comp Neurol 1992, 32 I:555-575.

20. Lavallée P, Urbain N, Dufresne C, Bokor H, Acsády L, Deschênes M: Feedforward inhibitory control of sensory information in higher-order thalamic nuclei. J Neurosci 2005 25(33):7489-7498.

21. Trageser JC, Burke KA, Masri RM, Li Y, Sellers L, Keller A: Statedependent gating of sensory inputs by zona incerta. J Neurophysiol 2006, 96:1456-1463.

22. Trageser JC, Keller A: Reducing the uncertainty: Gating of peripheral inputs by zona incerta. J Neurosci 2004, 24:89||-89|5

23. Huerta M, Frankfurter A, Harting J: The trigeminocolliculuar projection in the cat: patch-like endings withing the intermediate gray. Brain Research 198I, 221:1-13.

24. Mana S, Chevalier G: Honeycomb-like structure of the intermediate layers of the rat superior colliclulus: afferent and efferent connections. Neuroscience 200I, 103(3):673-693.

25. Mountcastle VB, Talbot WH, Sakata H, Hyvarinen J: Cortical neuronal mechanisms in flutter-vibration studied in unanesthetized monkeys. Neuronal periodicity and frequency discrimination. J Neurophysiol 1969, 32:452-484.

26. Simons DJ: Response properties of vibrissa units in rat SI somatosensory neocortex. J Neurophysiol 1978, 41:798-820.

27. Bruno RM, Simons DJ: Feedforward mechanisms of excitatory and inhibitory cortical receptive fields. I Neurosci 2002 22(24): $10966-10975$.
28. Kwegyir-Afful EE, Keller A: Response properties of whisker related neurons in rat second somatosensory cortex. J Neurophysiol 2004, 92:2083-2092.

29. Lee P, Hall WC: An in vitro study of horizontal connections in the intermediate layer of the superior colliculus. I Neurosci 2006, 26(I 8):4763-4768.

30. Simons DJ, Carvell GE: Thalamocortical response transformation in rat vibrissa/barrel system. I Neurophysiol 1989, 6I(2):3II-330.

31. Kyriazi HT, Carvell GE, Simons DJ: OFF response transformations in the whisker/barrel system. J Neurophysiol 1994, 72(I):392-40I.

32. Lichtenstein SH, Carvell GE, Simons DJ: Responses of rat trigeminal ganglion neurons to movements of vibrissae in different directions. Somatosens Mot Res 1990, 7(I):47-65.

33. Hartings JA, Temereanca S, Simons DJ: High responsiveness and direction sensitivity of neurons in the rat thalamic reticular nucleus to vibrissa deflections. I Neurophysiol 2000, 83(5):279|-280|.

34. Minnery BS, Simons DJ: Response properties of whisker-associated trigeminothalamic neurons in rat nucleus principalis. $J$ Neurophysiol 2003, 89(I):40-56.

35. Lee $\mathrm{SH}$, Simons $\mathrm{D}$ : Angular tuning and velocity sensitivity in different neuron classes within layer 4 of rat barrel cortex. $J$ Neurophysiol 2004, 9 I(I):223-229.

36. Hartings JA, Simons DJ: Inhibition suppresses transmission of tonic vibrissa-evoked activity in the rat ventrobasal thalamus. J Neurosci 2000, 20(19):RCI00.

37. Timofeeva $E$, Lavallee $P$, Arsenault $D$, Deschênes $M$ : The synthesis of multi-whisker receptive fields in subcortical stations of the vibrissa system. I Neurophysiol 2004, 91:1510-15I5

38. Masri RM, Trageser JC, Bezdudnaya T, Li Y, Keller A: Cholinergic regulation of the posterior medial thalamic nucleus. I Neurophysiol 2006, 96:2265-2273.

39. Diamond ME, Armstrong-James M, Ebner FF: Somatic sensory responses in the rostral sector of the posterior group (POm) and in the ventral posterior medial nucleus (VPM) of the rat thalamus. J Comp Neurol 1992, 3 I 8(4):462-476.

40. Friedberg MH, Lee SM, Ebner FF: Modulation of receptive field properties of thalamic somatosensory neurons by the depth of anesthesia. J Neurophysiol 1999, 8 I (5):2243-2252.

4I. Armstrong-James M, George MJ: Influence of anesthesia on spontaneous activity and receptive field size of single units in rat Sm I neocortex. Exp Neurol 1988, 99:369-387.

42. Furuta T, Nakamura K, Deschenes M: Angular tuning bias of vibrissa-responsive cells in the paralemniscal pathway. J Neurosci 2006, 26(4I): 10548-10557.

43. Nicolelis MAL, Chapin JK: Spatiotemporal structure of somatosensory responses of many-neuron ensembles in the rat ventral posterior medial nucleus of the thalamus. J Neurosci 1994, I4(6):35II-3532.

44. Killackey H, Erzurumlu R: Trigeminal projections to the superior colliculus of the rat. J Comp Neurol I 98I, 20I(2):22 I-242.

45. Sosnik R, Haidarliu S, Ahissar E: Temporal frequency of whisker movement. I. Representations in brain stem and thalamus. Neurophysiol 200I, 86(I):339-353.

46. Armstrong-James M, Callahan CA: Thalamo-cortical processing of vibrissal information in the rat. II. Spatiotemporal convergence in the thalamic ventroposterior medial nucleus (VPm) and its relevance to generation of receptive fields of $S I$ cortical "barrel" neurones. J Comp Neurol I 991, 303(2):2 I I-224.

47. Bruce LL, McHaffie JG, Stein BE: The organization of trigeminotectal and trigeminothalamic neurons in rodents: a doublelabeling study with fluorescent dyes. I Comp Neurol 1987, 262(3):3।5-330.

48. Jacquin MF, Mooney RD, Rhoades RW: Morphology, response properties, and collateral projections of trigeminothalamic neurons in brainstem subnucleus interpolaris of rat. Exp Brain Res 1986, 6 I(3):457-468. 